Diabetologia (1996) 39: 161-165

\title{
Loss of regulation by sympathetic hepatic nerves of liver metabolism and haemodynamics in chronically streptozotocin-diabetic rats
}

\author{
F. Stümpel ${ }^{1}$, T. Kucera ${ }^{1}$, R. Bazotte ${ }^{2}$, G. P. Püschel ${ }^{1}$ \\ ${ }^{1}$ Institut für Biochemie und Molekulare Zellbiologie, Georg-August-Universität, Göttingen, Germany \\ ${ }^{2}$ Departamento de Farmacologia e Farmácia, Universidade Estadual Maringá, Maringá, Paraná, Brasil
}

\begin{abstract}
Summary The consequences of autonomic diabetic neuropathy, a common complication of chronic diabetes mellitus, have been studied mainly with regard to heart and stomach function. Since the autonomic nervous system also regulates liver carbohydrate metabolism and haemodynamics via hepatic nerves, it was the purpose of this study to examine the function of hepatic nerves in chronically diabetic rats. Diabetes was induced by i.p. injection of streptozotocin. Rat livers were perfused via both portal vein and hepatic artery. Hepatic nerves were stimulated for 2 min using a platinum electrode placed around the portal vein and the hepatic artery; in an additional stimulation phase noradrenaline was infused into the portal vein. Stimulation of hepatic nerves as well as portal noradrenaline infusion increased hepatic glucose output and reduced flow in control and in acutely (48-h) diabetic animals, which still had almost normal glycogen content. In addition stimulation also
\end{abstract}

caused an overflow of noradrenaline into the caval vein. However, nerve stimulation neither increased glucose output nor decreased flow in 4-month diabetic rats. In these rats noradrenaline overflow was nearly completely abolished and hepatic glycogen content was markedly depleted. Portal noradrenaline infusion in chronically diabetic rats reduced flow to a similar extent as in controls, yet the increase in glucose output was diminished. The lack of nerve stimulation-dependent glucose output, flow reduction and noradrenaline overflow is indicative of a profound loss of function of hepatic autonomic nerves in chronically diabetic rats. [Diabetologia (1996) 39: 161-165]

Key words Isolated perfused liver, streptozotocin-induced diabetes, autonomic neuropathy, hepatic nerves, liver haemodynamics, glucose metabolism.
Neuropathy, like microangiopathy and retinopathy, is one of the most common late complications of diabetes mellitus $[1,2]$. However, the reported prevalence of diabetic neuropathy varies considerably between 10 and $100 \%$ in different studies [3]. In a recent standardized multicentre study a prevalence of neuropathy of $10-35 \%$ in insulin-dependent diabetic

Received: 19 April 1995 and in revised form 2 August 1995

Corresponding author: Dr. F. Stümpel, Institut für Biochemie und Molekulare Zellbiologie, Humboldtallee 23, D-37073 Göttingen, Germany

Abbreviations: IDDM, Insulin-dependent diabetes mellitus; NIDDM, non-insulin-dependent diabetes mellitus; IVC, inferior vena cava; BSA, bovine serum albumin.
(IDDM) patients and $25 \%$ in non-insulin-dependent diabetic NIDDM patients was found [3]. The main site for diabetic neuropathy is the sensory system, leading to hypaesthesia or hyperaesthesia and dysaesthesia whereas the motor nerves are only rarely affected. Diabetic neuropathy can also affect the autonomic nervous system. The prevalence of diabetic autonomic neuropathy varies depending on the criteria used for diagnosis. Only $0-10 \%$ of all patients complain of symptoms, excepting impotence, which can be seen in about $50 \%$ of all male patients. However, upon clinical examination autonomic neuropathy can be found in 17-22\% of patients with IDDM or NIDDM [3]. The best-studied manifestations of autonomic neuropathy are of cardiovascular (postural hypotension, cardiac arrhythmia, loss of respira- 
tory arrhythmia), gastrointestinal (gastric hypomotility, intestinal hypermotility) and genitourinary (bladder atony, male impotence) origin.

Hypoglycaemia unawareness and the lack of hyperglycaemic reflexes to hypoglycaemia are common complications in IDDM patients under intensive insulin therapy $[4,5]$. Patients do not perceive their hypoglycaemic state and they respond to hypoglycaemia with an attenuated increase in circulating catecholamine and glucagon levels $[6,7]$ that in turn could lead to mobilization of glucose from the liver by glycogenolysis and gluconeogenesis. Since the normal release of adrenaline in response to hypoglycaemia is blunted in these patients, autonomic neuropathy has been implicated as one possible cause of hypoglycaemia unawareness [7]. However, its role is still unclear, since this symptom can be found frequently without any other sign of autonomic neuropathy [8].

Liver metabolism and haemodynamics are regulated not only by circulating hormones but also by hepatic nerves [9]. Sympathetic and parasympathetic fibres enter the liver at the hilus as a plexus around the portal vein and hepatic artery. Central (ventromedial hypothalamus) [10,11] or peripheral ([hepatic nerves around the portal vein and hepatic artery) [12] stimulation of sympathetic hepatic nerves has been shown to increase hepatic glucose output in rats $[10,12]$, rabbits [13], dogs [14] and man [15]. This nerve stimulation-dependent increase in glucose output is mediated by $\alpha_{1}$-receptors [16] and is preceded by an overflow of noradrenaline into the hepatic vein [12]. The nerve stimulation-dependent increase in hepatic glucose output has been discussed as a possible means of supplying glucose for the body $[9,17]$, for example on acute stress such as acute haemorrhage [18]. The current study showed that this function of sympathetic hepatic nerves was severely impaired in chronically streptozotocin-diabetic rats with signs of profound diabetic neuropathy.

\section{Materials and methods}

Materials. All chemicals were of reagent grade and from commercial sources: enzymes, l-noradrenaline and bovine serum albumin (BSA) were purchased from Boehringer (Mannheim, Germany), streptozotocin from Sigma (Deisenhofen, Germany).

Animals. Male Wistar rats were obtained from Harlan-Winkelmann (Borchen, Germany). At least 1 week before the experiments they were subjected to a 12 -h day-night rhythm $(07.00$ 19.00 hours) with free access to food and water (standard diet of Ssniff, Soest, Germany). All perfusion experiments were started at 09.00 hours.

To induce diabetes, rats (weighing $100-120 \mathrm{~g}$ in the chronic diabetes group, $240-260 \mathrm{~g}$ in the acute diabetes group) were starved for $24 \mathrm{~h}$ and streptozotocin $(50 \mathrm{mg} / \mathrm{kg}$, dissolved in $0.05 \mathrm{~mol} / 1$ sodium citrate, $\mathrm{pH} 4.5$ ) was injected i. p.
Treatment of the animals followed the German Law on the Protection of Animals and was performed with permission of the state animal welfare committee.

Liver perfusion. The liver was perfused in situ without recirculation via both the portal vein and the hepatic artery $[19,20]$. The perfusion medium was an erythrocyte-free Krebs-Henseleit-bicarbonate buffer containing $5 \mathrm{mmol} / \mathrm{lglucose}, 2 \mathrm{mmol} / \mathrm{l}$ lactate, $0.2 \mathrm{mmol} / 1$ pyruvate and $0.5 \% \mathrm{BSA}$. The medium was equilibrated with $\mathrm{O}_{2}: \mathrm{CO}_{2}(19: 1)$.

Animals were anaesthetised by i.p. injection of pentobarbital $(60 \mathrm{mg} / \mathrm{kg})$. The abdomen was opened by a midline incision and heparin (50 IU) was injected into the inferior vena cava (IVC) to avoid coagulation. Blood and urine samples were drawn by puncturing the femoral artery and the urinary bladder, respectively. Immediately after the rat had been killed by an i. v. overdose of pentobarbital a catheter was introduced into the portal vein, the IVC was incised longitudinally and the liver perfusion started via the portal vein with a pressure of $10 \mathrm{mmHg}(1.33 \mathrm{kPa})$ and a flow rate of about $3.3 \mathrm{ml}$ $\min ^{-1} \cdot \mathrm{g}^{-1}$ organ weight. The position of the portal catheter was corrected in order to maintain sufficient space for placing an electrode around the portal vein and hepatic artery for nerve stimulation and then fixed by closing the ligature. Splenectomy and gastrectomy were performed following ligation of their vessels and ligation of the oesophagus and pylorus. The gastroduodenal artery and radix mesenterii were ligated and the intestine was removed. Connective tissue was removed from the aorta and common hepatic artery and the latter vessel was directly cannulated. At this stage additional perfusion via the artery was begun and a bivascular liver perfusion was established with a low pressure component via the portal vein $(10 \mathrm{mmHg}$ [1.33 kPa], $60-80 \%$ of total flow) and a high pressure component via the hepatic artery $(60-70 \mathrm{mmHg}[7.89$ $9.31 \mathrm{kPa}$ ], $20-40 \%$ of total flow) resulting in a total flow of $4 \mathrm{ml} \cdot \mathrm{min}^{-1} \cdot \mathrm{g}^{-1}$ organ weight. An outflow catheter was fixed with ligatures in the IVC with the tip placed at the inflow of the hepatic veins into the IVC. Finally the thorax was opened and the IVC and aorta were ligated. Experiments were started following a pre-perfusion period of $20 \mathrm{~min}$.

Nerve stimulation and infusion of noradrenaline. Since the hepatic nerves enter the liver via the anterior plexus around the hepatic artery and the posterior plexus around the portal vein [21], hepatic nerves were stimulated with a bipolar platinum wire electrode placed around both the portal vein and the hepatic artery $(20 \mathrm{~Hz}, 20 \mathrm{~V}, 2 \mathrm{~ms})$. Noradrenaline was dissolved in $154 \mathrm{mmol} / 1 \mathrm{NaCl}$ containing $100 \mathrm{mmol} / \mathrm{l} \mathrm{L}$ ascorbic acid and then diluted in the perfusion buffer containing $0.5 \% \mathrm{BSA}$ to an appropriate sinusoidal concentration of $1 \mu \mathrm{mol} / \mathrm{l}$.

Determination of metabolites and noradrenaline. Perfusion buffer samples were taken consecutively in 1-min intervals and directly chilled on ice. Metabolite concentrations were measured with standard enzymatic techniques: glucose was determined with glucose dehydrogenase (Merck glucose system, Merck KGaA, Darmstadt, Germany) [22] and lactate with lactate dehydrogenase and alanine aminotransferase [23]. Noradrenaline was quantitated electrochemically after separation by HPLC using the Waters system (Millipore GmbH Waters, Eschborn, Germany) [24]. Blood and urine glucose concentrations were determined with a Beckman glucose analyzer 2 (Beckmann, Munich, Germany) with the glucose oxidase method [25]. Urine samples were centrifuged and adequately diluted with Beckman dilution solution before glucose determination. 
Table 1. Blood glucose level, urine glucose level, liver glycogen content and body weight of non-diabetic, 4-months streptozotocin-diabetic and 48-h streptozotocin-diabetic rats

\begin{tabular}{|c|c|c|c|}
\hline & Control & $\begin{array}{l}\text { Diabetes } \\
4 \text { months }\end{array}$ & $\begin{array}{l}\text { Diabetes } \\
48 \mathrm{~h}\end{array}$ \\
\hline $\begin{array}{l}\text { Blood glucose } \\
\text { level (mmol/l) }\end{array}$ & $8.5 \pm 2.3$ & $29.6 \pm 6.2^{\mathrm{a}}$ & $24.6 \pm 4.8^{\mathrm{a}}$ \\
\hline $\begin{array}{l}\text { Urine glucose } \\
\text { level (mmol/l) }\end{array}$ & $\begin{array}{l}\text { not } \\
\text { detectable }\end{array}$ & $63.4 \pm 4.2$ & $54.7 \pm 0.2$ \\
\hline $\begin{array}{l}\text { Liver glycogen } \\
\text { ( } \mu \text { mol glycosyl } \\
\left.\text { units } \cdot \mathrm{g}^{-1}\right)\end{array}$ & $131.6 \pm 34.2$ & $11.8 \pm 0.9^{\mathrm{a}}$ & $135.2 \pm 11.8^{\mathrm{b}}$ \\
\hline Body weight (g) & $309.6 \pm 16.8$ & $300.4 \pm 21.9^{\mathrm{b}}$ & $276.5 \pm 22.5^{b}$ \\
\hline
\end{tabular}

The values are means \pm SEM of eight (control), four (chronic diabetic) and four (acute diabetic) experiments

${ }^{\mathrm{a}} p<0.025$ vs control; ${ }^{b}$ not significant vs control

Determination of liver glycogen content. Immediately after the end of the experiments tissue samples of the livers were homogenized for $30 \mathrm{~s}$ with an Ultra-Turrax (Kunkel, Staufen, Germany) in perchloric acid $(1 \mathrm{~mol} / \mathrm{l})$. Glycogen was hydrolysed with aminoglucosidase at $\mathrm{pH} 4.6$ and free glucose was measured with glucose dehydrogenase [22]. Liver glycogen content was expressed as umol glucosyl units $\cdot$ g liver $^{-1}$.

Measurement of perfusion flow rates. Total perfusion flow was quantified by fractionating the effluente into calibrated tubes. The portal flow was measured with an SMS-302 electromagnetic flow meter (Hellige, Freiburg, Germany) placed into the portal inflow. The arterial flow was calculated as the difference between total and portal flow.

\section{Statistical analysis}

All results are presented as mean $\pm S E M$ for the indicated number of observations. Data were analysed by Student's $t$ test for unpaired data. Differences were considered significant at $p<0.05$.

\section{Results}

In the present study the effect of electrical stimulation of hepatic nerves was analysed in the livers of rats with streptozotocin-induced diabetes perfused in situ via both the hepatic artery and portal vein to evaluate the possible impairment by autonomic diabetic neuropathy of hepatic nerve function in three experimental groups. The first group consisted of weight-matched non-diabetic rats, the second one of rats made diabetic for about 4 months and the third group was composed of acutely 48 -h streptozotocintreated diabetic rats to exclude a possible neurotoxic effect of streptozotocin.

Induction of diabetes by streptozotocin. Following the i.p. streptozotocin injection all treated rats developed clinical signs of hyperglycaemia (e.g. polydipsia and polyuria) within the following $36-60 \mathrm{~h}$. In the 4months diabetic group only rats with blood glucose concentrations of more than $20 \mathrm{mmol} / 1$ were used for the experiments. At the time of the experiments both streptozotocin-treated groups, the 4-months diabetic rats and the 48-h diabetic rats showed pathological blood and urine glucose concentrations (Table 1). All rats that had been diabetic for 4 months also showed signs of severe complications of chronic diabetes (e.g. peripheral neuropathy, enlarged bowels and urinary bladders, as well as cataracts). Glycogen content of livers of non-diabetic rats was comparable with previous results [25] (Table 1). In livers of 4months streptozotocin-diabetic rats hardly any glycogen could be detected, in contrast livers of 48-h streptozotocin-diabetic rats contained normal amounts of glycogen (Table 1).

Effects of hepatic nerve stimulation and portal noradrenaline infusion on hepatic metabolism and haemodynamics. Hepatic nerves were stimulated $(20 \mathrm{~Hz}$, $20 \mathrm{~V}, 2 \mathrm{~ms}$ ) (Fig. 1) and as a second stimulus noradrenaline $(1 \mu \mathrm{mol} / 1)$ was infused into the portal vein for 2-min periods (Fig. 1). Nerve stimulation increased glucose output and shifted lactate uptake to output in livers of non-diabetic rats (Fig.1A, upper panel). The metabolic alterations reached a maximum (peak value) at about 3 min after the onset of stimulation. Total, venous and arterial flow were markedly decreased with maximal effects after 2 min (Fig.1A, lower panel). Quantity and time course of glucose and lactate output and haemodynamic changes were comparable with previous results $[26,27]$. In contrast, in livers of 4-months streptozotocin-diabetic rats the increase in glucose output and shift of lactate uptake to output following hepatic nerve stimulation was nearly abolished and a decrease in total, portal and arterial flow could not be measured (Fig. 1B). Nerve stimulation in the 48-h streptozotocin-diabetic rats caused nearly the same metabolic and haemodynamic changes as in non-diabetic rats. However, basal glucose output and lactate uptake were higher than in controls, so that lactate uptake was nearly reduced but not shifted to output (Fig. 1C).

Infusion of noradrenaline into the portal vein of livers of non-diabetic rats increased glucose and lactate output with a peak value at about 4 min after the onset of infusion and a decrease in total and portal flow with a maximum effect at 2 min (Fig.1A). Noradrenaline-elicited glucose and lactate output from livers of 4-months and 48-h streptozotocin-diabetic rats, respectively, was only slightly smaller than from livers of non-diabetic rats; the haemodynamic changes were similar (Fig.1B,C).

Noradrenaline overflow following hepatic nerve stimulation. Stimulation of hepatic nerves of livers of nondiabetic rats resulted in an increase in noradrena- 


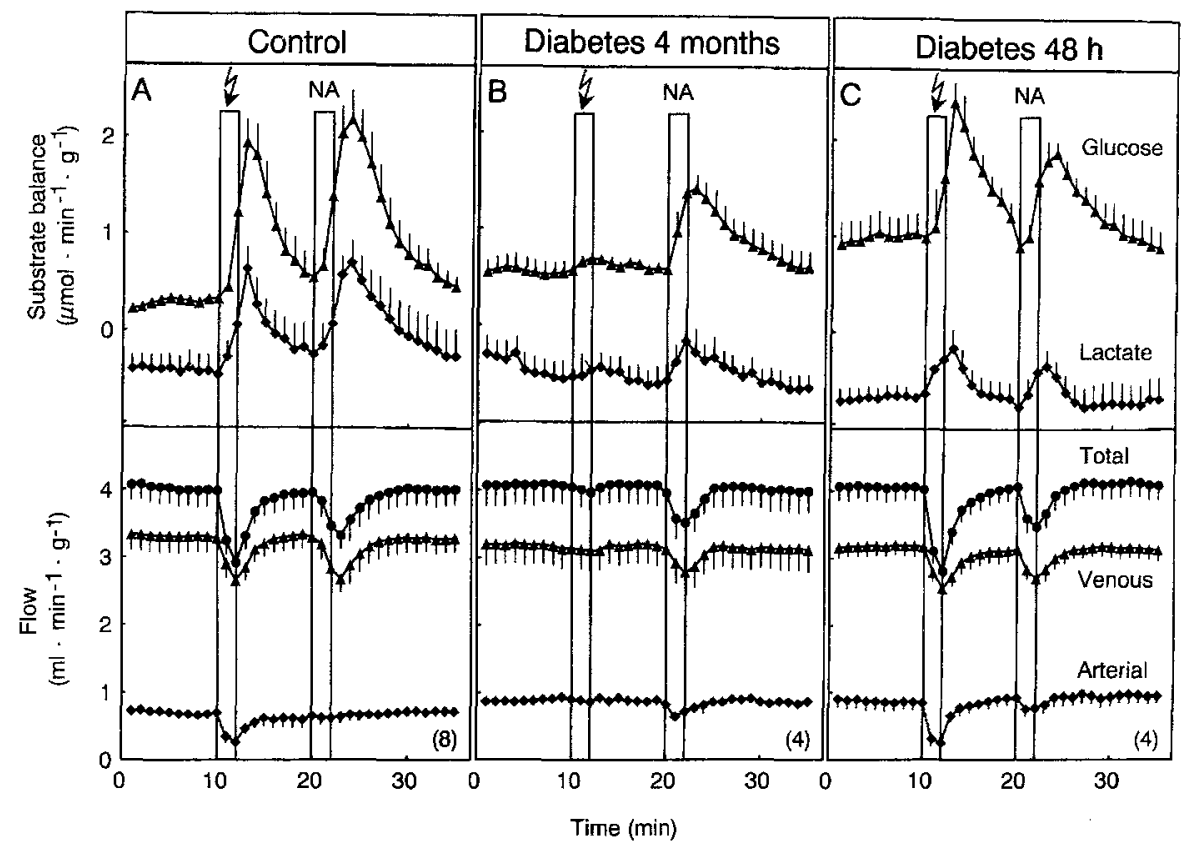

Fig. 1. Effects of neurostimulation and portal noradrenaline infusion on hepatic glucose and lactate metabolism and portal and arterial flow in non-diabetic, 4months streptozotocin-diabetic and 48-h streptozotocin-diabetic rats. Diabetes was induced as described in Methods. The hepatic nerves were stimulated during liver perfusion as indicated during min 11 and $12(20 \mathrm{~Hz}, 20 \mathrm{~V}, 2 \mathrm{~ms})$ by placing an electrode in the hilus around the portal vein and hepatic artery. Noradrenaline (NA) was infused into the portal vein during min 21 and 22 of perfusion with a final concentration of $1 \mu \mathrm{mol} / 1$. Values are means $\pm \mathrm{SEM}$ of the number of experiments given in parentheses
Table 2. Noradrenaline overflow following neurostimulation of livers of non-diabetic, 4-months streptozotocin-diabetic and 48-h streptozotocin-diabetic rats

\begin{tabular}{llll}
\hline $\begin{array}{l}\text { Noradrenaline } \\
\left(\mathrm{pmol} \cdot \mathrm{min}^{-1} \cdot \mathrm{g}^{-1}\right)\end{array}$ & Control & $\begin{array}{l}\text { Diabetes } \\
4 \text { months }\end{array}$ & Diabetes $48 \mathrm{~h}$ \\
\hline $\begin{array}{l}\text { Basal } \\
\text { Peak following } \\
\text { neurostimulation }\end{array}$ & $21.65 \pm 0.13$ & $4.04 \pm 0.13^{\mathrm{a}}$ & $3.83 \pm 0.49^{\mathrm{a}}$ \\
\hline
\end{tabular}

The values are means \pm SEM of eight (control), four (chronic diabetic) and four (acute diabetic) experiments

${ }^{\text {a }}$ Not significant different from control; ${ }^{b}$ significantly different from control, $p<0.025 ;{ }^{\circ}$ significantly higher than basal, $p<$ 0.025

line overflow into the hepatic veins (basal $3.65 \pm 0.13$ $\mathrm{pmol} \cdot \mathrm{min}^{-1} \cdot \mathrm{g}^{-1}$, peak following nerve stimulation $21.20 \pm 3.90 \mathrm{pmol} \cdot \mathrm{min}^{-1} \cdot \mathrm{g}^{-1}$ ) with the nadir at $2 \mathrm{~min}$ (Table 2). The increase in noradrenaline overflow was markedly reduced in livers of 4-months diabetic rats but still normal in the 48 -h diabetic rats (Table 2 ).

\section{Discussion}

The hepatic nerve stimulation-dependent increase in glucose output was abolished in 4-months diabetic rats. By contrast, noradrenaline-elicited increase in glucose output was only moderately reduced in chronically diabetic rats. The lack of an effect of electrical stimulation on glucose output could be due to the drastic reduction of glycogen stores or to a loss of function of hepatic nerves. In a previous study in [28] starved rats with widely depleted glycogen stores such as in the chronically diabetic animals, nerve stimulation-dependent glucose output was reduced to about $30 \%$ of the values in fed rats; it was due equally to glycogenolysis (50\%) and gluconeogenesis $(50 \%)$. In the current study circulating noradrenaline, which is also the neurotransmitter involved in nerve stimulation-dependent glucose output, elicited a release of glucose from livers of chronically diabetic rats, which was about $50 \%$ of the values of normal rats. Thus, the loss of nerve stimulation-dependent glucose output in chronically diabetic rats was not the result of the depletion of glycogen stores but of the destruction of hepatic nerves.

Nerve stimulation-dependent reduction of flow, both through the hepatic artery and the portal vein, was abolished in 4-months diabetic rats, whereas the noradrenaline-elicited flow reduction was identical in extent and kinetics to non-diabetic controls. Therefore, the lack of a nerve stimulation-dependent flow reduction in chronically diabetic rats is not due to an impaired contractile function of the vascular system but to the loss of function of sympathetic hepatic nerves.

The most direct measure of function of hepatic sympathetic nerves is the nerve stimulation-dependent noradrenaline overflow. Noradrenaline, released from the varicosities of sympathetic hepatic nerves upon nerve stimulation is eliminated from the synapses partially by re-uptake into the nerve endings, partially by diffusion into the circulation. This latter fraction can be detected post-hepatically in the perfusate [12]. In non-diabetic controls, as in acutely diabetic rats the noradrenaline overflow increased about sixfold over basal shortly after onset of nerve stimulation. By contrast, the nerve stimulation-dependent noradrenaline overflow in chronically diabetic rats was almost entirely abolished. The loss of nerve stimulation-dependent noradrenaline overflow in chroni- 
cally diabetic rats could not be ascribed to a toxic side effect of streptozotocin, since the noradrenaline overflow was not impaired in acutely diabetic rats, which had received the same dose of streptozotocin.

Common complications in IDDM patients with good euglycaemic control due to insulin treatment are hypoglycaemia and hypoglycaemia unawareness, in that patients do not react with an increase in glucagon and catecholamine levels in response to hypoglycaemia $[6,7]$. The aetiology of this phenomenon is controversial and diabetic sympathetic neuropathy has been implicated in its development.

Hepatic glucose output in response to hypoglycaemia cannot be regulated by circulating hormones alone but also, more directly, by the action of sympathetic hepatic nerves [9]. Thus, diabetic sympathetic neuropathy affecting hepatic nerves, would deprive the chronically diabetic patient, with reduced capacity to respond to hypoglycaemia with an adequate increase in circulating catecholamines and glucagon, of the last means to respond to hypoglycaemia with a mobilization of hepatic glucose stores. Consequently, patients whose hepatic sympathetic nerve function is impaired due to diabetic neuropathy could be even more prone to hypoglycaemic complications than patients without a loss of function of sympathetic hepatic nerves.

Acknowledgements. This work was supported by the Deutsche Forschungsgemeinschaft through the Sonderforschungsbereich 402 Teilprojekt B3 and B6 as well as by a travel research grant of CNPq, Brasil, to GP Püschel. The authors wish to thank Prof. Jungermann for his invaluable advise and his discussion during the preparation of the manuscript.

\section{References}

1. Horowitz SH (1993) Diabetic neuropathy. Clin Orth Rel Res 296: 78-85

2. Vinik A, Holland M, Le Beaux JM, Liuzzi FJ, Stransberry KB, Colen LB (1992) Diabetic neuropathies. Diabetes Care 15: 1926-1975

3. Ziegler D, Gries FA, Spüler M, Lessmann F, DiaCAN Multicenter Study Group (1993) The epidemiology of diabetic Neuropathy. Diabet Med 10: 82S-86S

4. Meijer E, Hoekstra JBL, Erkelens DW (1994) Hypoglycaemia unawareness. Presse Med 23: 623-627

5. The DCCT Research Group (1993) The effect of intensive treatment of diabetes on the development and progression of long-term complications in insulin-dependent diabetes mellitus. N Engl J Med 329: 977-986

6. Simonson DC, Tamborlane WV, DeFronzo RA, Sherwin RS (1985) Intensive insulin therapy reduces counterregulatory hormone responses to hypoglycaemia in patients with type I diabetes. Ann Rev Intern Med 103: 184-190

7. Webb SM, Fernandez Castaner M (1985) Glucose counterregulation in diabetic autonomic neuropathy. Clin Physiol 5[Suppl 5]:66-71

8. Hoeldtke RD, Boden G (1994) Epinephrine secretion, hypoglycaemia unawareness, and diabetic autonomic neuropathy. Ann Int Med 120: 512-517
9. Gardemann A, Püschel GP, Jungermann K (1992) Nervous control of liver metabolism and haemodynamics. Eur J Biochem 207: 399-411

10. Shimazu $\mathrm{T}$ (1987) Neuronal regulation of hepatic glucose metabolism in mammals. Diabetes Metab Rev 3: 185-206

11. Iwai M, Shimazu T (1988) Effects of ventromedial and lateral hypothalamic stimulation on chemically-induced liver injury in rats. Life Sci 42: 1833-1840

12. Hartmann H, Beckh K, Jungermann K (1982) Direct control of glycogen metabolism in the perfused rat liver by the sympathetic innervation. Eur J Biochem 123: 521-526

13. Shimazu T, Usami M (1982) Further studies on the mechanism of phosphorylase activation in rabbit liver in response to splanchnic nerve stimulation.J Physiol 329: 231-242

14. Edwards AV (1971) The glycogenolytic response to stimulation of the splanchnic nerves in adrenalectomized calves, sheep, dogs, cats and pigs. J Physiol 213: 741-753

15. Nobin A, Falck B, Ingemanson S, Järhuld J, Rosengren E (1977) Organization and function of the sympathetic innervation of the human liver. Acta Physiol Scand [Suppl] 452: 103-106

16. Ulken V, Püschel GP, Jungermann K (1991) Increase in glucose and lactate output and perfusion resistance by stimulation of hepatic nerves in isolated perfused rat liver: role of a1-, a2-, $\beta 1$ - and $\beta 2$-receptors. Biol Chem Hoppe Seyler 372: 401-409

17. Yamaguchi N (1992) Sympathoadrenal system in neuroendocrine control of glucose: mechanism involved in the liver, pancreas, and adrenal gland under hemorrhagic and hypoglycemic stress. Can J Physiol Pharmacol 70: 167-206

18. Lautt WW, Dwan PD, Singh RR (1982) Control of the hyperglycemic response to haemorrhage in cats. Can J Physiol Pharmacol 60: 1618-1623

19. Gardemann A, Strulik H, Jungermann K (1987) Nervous control of glycogenolysis and blood flow in arterially and portally perfused rat liver. Am J Physiol 253:E238-E245

20. Gardemann A, Jahns U, Jungermann K (1991) Control of glycogenolysis and blood flow by arterial and portal noradrenaline in perfused liver. Am J Physiol 260:E762-E771

21. Metz W, Forssmann WG (1980) Innervation of the liver in guinea pig and rat. Anat Embryol 160: 239-252

22. Banauch D, Brümmer W, Ebeling W et al. (1975) Eine Glucose Dehydrogenase für die Glucose-Bestimmung in Körperflüssigkeiten. Z Klin Chem Klin Biochem 13: 101-107

23. Noll F (1974) Lactat-Bestimmung mit LDH, GPT und NAD. In: Bergmeyer HU (ed) Methoden der enzymatischen Analyse. Chemie, Weinheim, pp 1521-1525

24. Scott MC, Elchisak MA (1987) Direct measurement of dopamine $\mathrm{O}$-sulphate in plasma and cerebral fluid. J Chromatogr 413: 17-23

25. Gibson OH, Swoboda BEP, Massey V (1964) Kinetics and mechanism of action of glucose oxidase. J Biol Chem 239: 3927-3934

26. Miura H, Gardemann A, Rosa J, Jungermann K (1992) Inhibition by noradrenaline and adrenaline of the increase in glucose and lactate output and decrease in flow after sympathetic nerve stimulation in perfused rat liver: possible involvement of protein kinase C. Hepatology 15: 477-484

27. Seseke F, Gardemann A, Jungermann K (1992) Signal propagation via gap junctions, a key step in the regulation of liver metabolism by the sympathetic hepatic nerves. FEBS Lett 301: 265-270

28. Beuers U, Jungermann K (1990) Relative contribution of glycogenolysis and gluconeogenesis to basal, glucagonand nerve stimulation-dependent glucose output in the perfused liver from fed and fasted rats. Biochem Int 21: 405415 